\title{
Syringe needle skull penetration reduces brain injuries and secondary inflammation following intracerebral neural stem cell transplantation
}

\author{
MOU GAO ${ }^{1,2 *}$, QIN DONG ${ }^{3 *}$, HONGTIAN ZHANG $^{2}$, YANG YANG $^{2}$, \\ JIANWEI ZHU ${ }^{2}$, ZHIJUN YANG ${ }^{2}$, MINHUI XU ${ }^{1}$ and RUXIANG XU ${ }^{2}$ \\ ${ }^{1}$ Department of Neurosurgery, Daping Hospital, Third Military Medical University, Chongqing 400042; \\ ${ }^{2}$ Affiliated Bayi Brain Hospital, PLA Army General Hospital, Beijing 100700; \\ ${ }^{3}$ Department of Neurology, Fu Xing Hospital, Capital Medical University, Beijing 100038, P.R. China
}

Received October 23, 2015; Accepted November 25, 2016

DOI: $10.3892 /$ etm.2017.4054

\begin{abstract}
Intracerebral neural stem cell (NSC) transplantation is beneficial for delivering stem cell grafts effectively, however, this approach may subsequently result in brain injury and secondary inflammation. To reduce the risk of promoting brain injury and secondary inflammation, two methods were compared in the present study. Murine skulls were penetrated using a drill on the left side and a syringe needle on the right. Mice were randomly divided into three groups ( $n=84 /$ group): Group A, receiving NSCs in the left hemisphere and PBS in the right; group B, receiving NSCs in the right hemisphere and PBS in the left; and group C, receiving equal NSCs in both hemispheres. Murine brains were stained for morphological analysis and subsequent evaluation of infiltrated immune cells. ELISA was performed to detect neurotrophic and immunomodulatory factors in the brain. The findings indicated that brain injury and secondary inflammation in the left hemisphere were more severe than those in the right hemisphere, following NSC transplantation. In contrast to the left hemisphere, more neurotrophic factors but less pro-inflammatory cytokines were detected in the right hemisphere. In addition, increased levels of neurotrophic factors and interleukin (IL)-10 were observed in the NSC transplantation side when compared with
\end{abstract}

Correspondence to: Dr Minhui Xu, Department of Neurosurgery, Daping Hospital, Third Military Medical University, 10 Changjiang Road, Yuzhong, Chongqing 400042, P.R. China

E-mail: dpmhxu@126.com

Dr Ruxiang Xu, Affiliated Bayi Brain Hospital, PLA Army General Hospital, 5 Dongcheng, Beijing 100700, P.R. China

E-mail: jzprofxu@126.com

*Contributed equally

Key words: neural stem cell, transplantation, injury, inflammation, immune cell the PBS-treated hemispheres, although lower levels of IL-6 and tumor necrosis factor- $\alpha$ were detected. In conclusion, the present study indicated that syringe needle skull penetration vs. drill penetration is an improved method that reduces the risk of brain injury and secondary inflammation following intracerebral NSC transplantation. Furthermore, NSCs have the potential to modulate inflammation secondary to brain injuries.

\section{Introduction}

Transplanted neural stem cells (NSCs) are involved in the repair of central nervous system (CNS) disease, predominantly by replacing damaged cells, secreting neurotrophic factors and participating in immune regulation (1-3). Therapeutic efficacy is associated with the quantity and quality of grafted NSCs and is also largely dependent on the implantation approach. Intracerebral transplantation can directly deliver exogenous NSCs through the blood-brain barrier into the immune-privileged brain. However, this approach requires a hole to be drilled into skull, which is invasive and associated with risks. Previous studies have demonstrated that several methods may be utilized to reduce brain injuries during penetration of the skull, in particular microtransplantation was found to improve the function of grafts $(4,5)$; however, the mechanism remains unclear. Furthermore, inflammation secondary to brain injury during skull penetration also influences the survival and function of NSCs (4-6), although fewer studies focus on this issue.

Previous studies have elucidated the relationship between NSCs and immune cells, and identified that the immune system has a complex role in CNS degeneration and reconstruction $(6,7)$. For example, while infiltrated immune cells are considered to be pathological, some studies have demonstrated that they have protective and healing properties in the CNS $(8,9)$. However, inflammation involving multiple activated immune cells may aggravate brain injuries and constitute a temporary risk to NSC survival.

In the present study, the effects of transplantation approaches on brain injuries and secondary inflammation 
were investigated. Two skull penetration models in adult mice were constructed where mice received either syngeneic NSCs or PBS and brain injuries and secondary inflammation from 1 to 28 days after implantation was observed.

\section{Materials and methods}

Animals. A total of 252 healthy adult C57BL/6 (B6) mice (126 male and 126 female; age, 10-12 weeks), weighing 18-28 g (Vital River Laboratories, Beijing, China) were housed in a temperature- and humidity-controlled room (temperature at $21 \pm 2^{\circ} \mathrm{C}$ and relative humidity at $50 \pm 5 \%$ ) and maintained on a $12 \mathrm{~h}$ light/dark schedule with free access to food and water. All experimental procedures were approved by the Animal Care and Use Committee of Third Military Medical University (Chongqing, China) and were in compliance with the Guide for the Care and Use of Laboratory Animals published by the National Institutes of Health.

Cells. B6 NSCs were isolated from the forebrain region of 13.5-day-old murine embryos and collected by several cycles of $0.05 \%$ trypsin-EDTA (Invitrogen; Thermo Fisher Scientific, Inc., Waltham, MA, USA) digestion. Cells were plated at a density of $10^{5}-10^{6}$ cells $/ \mathrm{ml}$ and cultured in neural stem cell culture medium (NSCcm) which was composed of DMEM/F12 (1:1) supplemented with 2\% B27, $20 \mathrm{ng} / \mathrm{ml}$ basic fibroblast growth factor (bFGF) and $20 \mathrm{ng} / \mathrm{ml}$ epidermal growth factor (EGF) (all Invitrogen; Thermo Fisher Scientific, Inc.). For identification, NSCs were digested with Accutase (Invitrogen; Thermo Fisher Scientific, Inc.) and seeded on glass coverslips coated with poly-1-lysine (Sigma-Aldrich; Merck Millipore, Darmstadt, Germany) in a 24-well plate $\left(1 \times 10^{5}\right.$ cells per well) in NSCcm for 2-3 days. The self-renewal potential of NSCs was determined by morphological assessment and immunofluorescence staining with the NSC-specific markers Nestin and Sox2. For transplantation, NSCs were digested with accutase and washed with PBS. Cells were counted by trypan blue (Sigma-Aldrich; Merck Millipore) exclusion in a haemocytometer and the density of single cell suspension was adjusted accordingly. Cells were maintained on ice.

Transplantation experiment. Mice were anesthetized with $3.6 \%$ chloral hydrate $(10 \mathrm{ml} / \mathrm{kg}$; intraperitoneal injection; Sigma-Aldrich; Merck Millipore) and mounted using stereotaxic apparatus (Stoelting, Wood Dale, IL, USA). After shaving and cleaning the skin, a midline scalp incision was made using an 11 Swann scalpel and the parietal bone was exposed.

For the drilling method, an appropriate puncture point was selected on the left hemisphere, and the skull was penetrated by drilling a hole with a sterile drill (Slite P-500-3A; Ningbo Rising Trading Co., Ningbo, China) using a steel screw (diameter, $1 \mathrm{~mm}$ ) at the speed of 12,000 rpm. For skull penetration with a syringe needle, an appropriate puncture point was selected on the right hemisphere, and the skull was penetrated with a sterile $25-\mu \mathrm{l} 22 \mathrm{~S}$ Hamilton syringe. The syringe was withdrawn once through the skull.

Mice were randomly assigned to three groups ( $n=84 /$ group): Group A, receiving NSCs in the left hemisphere and the same volume of PBS in the right hemisphere; group B, receiving NSCs in the right hemisphere and PBS in the left hemisphere; and group C, receiving the equal amount of NSCs in the two hemispheres. Cell suspension or PBS was injected into the motor cortex $(4.5 \mathrm{~mm}$ anterior to the lambda suture, $1.0 \mathrm{~mm}$ lateral to the middle line and $2.5 \mathrm{~mm}$ under the dura) using different sterile $25-\mu 122 \mathrm{~S}$ Hamilton syringes. Each site received $5 \mu 1$ of cell suspension containing $5 \times 10^{5}$ cells or PBS at a speed of $0.5 \mu \mathrm{l} / \mathrm{min}$. Following injection for $\sim 5 \mathrm{~min}$, the syringe was slowly withdrawn. At 1, 2, 3, 7, 14, 21 and 28 days after transplantation, mice were sacrificed using a lethal dose of $10 \mathrm{ml} / \mathrm{kg}$ chloral hydrate and their brain tissues were harvested for morphological and molecular-biological analysis.

Morphological analysis. Brain tissues were postfixed in $4 \%$ paraformaldehyde in $0.1 \mathrm{M}$ PBS $(\mathrm{pH} 7.4)$ at $4^{\circ} \mathrm{C}$ overnight. Tissues were sectioned $(8 \mu \mathrm{m})$ on a cryostat (Leica CM1950; Leica Microsystems, Inc., Buffalo Grove, IL, USA) and mounted to an adhesion microscope slide. Pathological features were assessed using hematoxylin and eosin staining and viewed under a light microscope (DM2000; Leica Microsystems, Inc.) at x200 magnification.

Immunofluorescence analysis. Cultured cells and brain slices were blocked using $10 \%$ bovine serum albumin and $0.3 \%$ Triton $\mathrm{X}-100$ for $1 \mathrm{~h}$, and subsequently incubated overnight at $4^{\circ} \mathrm{C}$ with primary antibodies (Table I). Following washing in PBS, cells and slices were incubated for 1-2 h at room temperature with secondary antibodies (Table I). Following several washes, the nuclei were stained using DAPI Fluoromount-G (SouthernBiotech, Birmingham, AL, USA) and coverslips were mounted onto glass slides and analyzed using a fluorescent microscope (DM3000) or a confocal laser scanning microscope (TCS SP5 II; both Leica Microsystems, Inc.). The number of positive cells was manually counted at $\times 200$ magnification directly under the microscope and adjusted using image analysis software (Image Pro Plus 5.0; Media Cybernetics, Inc., Rockville, MD, USA). The ratio of positive cells was calculated as follows: Ratio of positive cells $=$ (number of positive cells/total number of cells) x $100 \%$.

ELISA. Brain tissues were respectively isolated from the two hemispheres and weighed. Protein was extracted from these brain tissues using radioimmunoprecipitation assay buffer (Sigma-Aldrich; Merck Millipore) supplemented with protease and phosphatase inhibitors (Fermentas; Thermo Fisher Scientific, Inc.). Protein concentration was determined using BCA assay (Thermo Fisher Scientific, Inc.). Mouse brain-derived neurotrophic factor (BDNF) glial cell line-derived neurotrophic factor (GDNF), neurotrophin-3 (NT-3), interleukin-6 (IL-6), interleukin (IL)-10 and tumor necrosis factor alpha (TNF- $\alpha$ ) were detected using ELISA kits. BDNF ELISA kit was purchased from Promega Corp., (Madison, WI, USA). GDNF ELISA kit was purchased from Santa Cruz Biotechnology Inc., (Dallas, TX, USA). NT-3 ELISA kit was purchased from Abcam, (Cambridge, MA, USA) and IL-6, IL-10 and TNF- $\alpha$ ELISA kits were purchased from Sigma-Aldrich (Merck Millipore). ELISA 
Table I. Antibodies used in the present study.

\begin{tabular}{|c|c|c|c|c|c|}
\hline Specificity & Host & Clone & Dilution & Detection & Catalogue no. \\
\hline Nestin $^{\mathrm{a}}$ & Mouse & Rat-401 & $1: 1,000$ & NSCs & MAB353 \\
\hline Sox $2^{b}$ & Goat & Y-17 & $1: 200$ & NSCs & sc- 17320 \\
\hline $\mathrm{CD}^{\mathrm{c}}$ & Rabbit & Polyclonal & $1: 200$ & $\mathrm{~T}$ cells & ab5690 \\
\hline CD11 $b^{c}$ & Rat & Monoclonal & $1: 200$ & Microglia/Macrophages & ab8878 \\
\hline $\mathrm{CD} 19^{\mathrm{d}}$ & Rat & $1 \mathrm{D} 3$ & $1: 200$ & B cells & LS-C107165 \\
\hline Mouse $\mathrm{IgG}^{\mathrm{e}}$ & Donkey & Polyclonal & $1: 1,000$ & Nestin & A-21203 \\
\hline Rat $\operatorname{IgG}^{\mathrm{e}}$ & Donkey & Polyclonal & $1: 1,000$ & $\mathrm{CD} 11 \mathrm{~b} / \mathrm{CD} 19$ & A-21209 \\
\hline Rabbit $\operatorname{IgG}^{\mathrm{e}}$ & Donkey & Polyclonal & $1: 1,000$ & $\mathrm{CD} 3$ & A-21207 \\
\hline Goat $\operatorname{IgG}^{\mathrm{e}}$ & Donkey & Polyclonal & $1: 1,000$ & Sox 2 & A-11055 \\
\hline
\end{tabular}

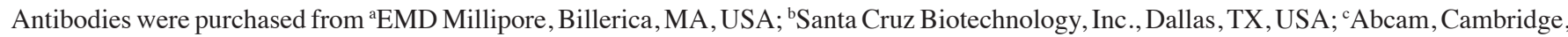
MA, USA; 'LifeSpan BioSciences, Inc., Seattle, WA, USA; and ${ }^{\mathrm{e}}$ Thermo Fisher Scientific, Inc., Waltham, MA, USA. CD, cluster of differentiation; IgG, immunoglobulin G; NSCs, neural stem cells.

was carried out in duplicate, according to the manufacturers' protocols.

Statistical analysis. The SPSS 17.0 statistical software (SPSS, Inc., Chicago, IL, USA) package was used for statistical analysis. Data were presented as mean \pm standard deviation. Student's t-test and one-way analysis of variance were used to determine statistical significance. $\mathrm{P}<0.05$ was considered to indicate a statistically significant difference.

\section{Results}

Identification of NSCs. B6 NSCs were isolated from the forebrain region of 13.5-day-old murine embryos and had self-renewal potential. These cells formed neurospheres that grew in culture medium in a state of suspension. The results of immunofluorescence staining indicated that these cells were strongly positive for Nestin and Sox2 (data not shown).

General information and morphological analysis. Macroscopic inspection showed obvious injuries and hemorrhage in the left hemisphere during the early stage of post-implantation for all the groups (data not shown). Wounds observed were irregular, unstable and varied within the drilling method group; whereas in the right hemisphere, wounds were small and exhibited a similar appearance to one another following syringe needle penetration. Histological examination of all groups indicated that a small amount of bleeding was observed in the first two days in the right hemisphere, which underwent syringe penetration. In contrast, the structure of the left hemisphere, which underwent drill penetration, was broken and exhibited extensive necrosis, infiltrated immune cells and hemorrhage. After one week, scar tissue formation was observed in all groups, and only a small number of neural cells were detected in the left hemisphere, whereas the contralateral cells were well-arranged. After 28 days, neural cells were rarely observed in the injured area, which was filled with massive scar tissue in the left hemisphere. In addition, brain injuries in the left hemisphere of mice receiving PBS treatment were notably worse than those receiving NSC transplantation.

Dynamic changes of infiltrated immune cells. To measure the extent of inflammation secondary to brain injury after implantation, the distribution of infiltrated immune cells, including microglia/macrophages [cluster of differentiation (CD)11b], T cells (CD3) and B cells (CD19) was observed. The results of group $C$ in the left and right hemispheres are presented in Fig. 1A and B, respectively. The number of immune cells was elevated in the left hemisphere; however, these cells were rarely detectable in the right hemisphere in all groups. As demonstrated in Fig. 1C-E, in group A, the three types of immune cells (CD11b, CD3 and CD19, respectively) increased rapidly in the first three days and remained at a high level for 2-3 weeks. In group B, the difference between the two hemispheres was the most obvious and the number of immune cells in the left hemisphere was the highest of the three groups. Only a small number of infiltrated immune cells were detected in group $\mathrm{C}$, the difference between the two hemispheres in this group was statistically significant $(\mathrm{P}<0.05)$. In addition, the number of immune cells in the part of the hemisphere receiving NSC transplantation was lower than that in the same side receiving PBS treatment $(\mathrm{P}<0.05)$.

Immune cells were predominantly distributed around the broken structure of the skull and were positively correlated with the degree of injury. An increase in the number of immune cells observed suggested that inflammation secondary to skull penetration using a drill was significantly more severe when compared with skull penetration using a syringe needle $(\mathrm{P}<0.05)$. The number of microglia and macrophages increased in the first week but the peak remained only for a short time (Fig. 1C). T cells were noticeable at the early stage following brain injury; however, the level of T cells did not change significantly throughout the study period (Fig. 1D). Compared with the other immune cells, the number of B cells was lower and the peak appeared later in three weeks $(\mathrm{P}<0.001$; Fig. 1E). In addition, the number of immune cells varied within the left hemisphere treatment group among all the groups (groups A-C). 
A
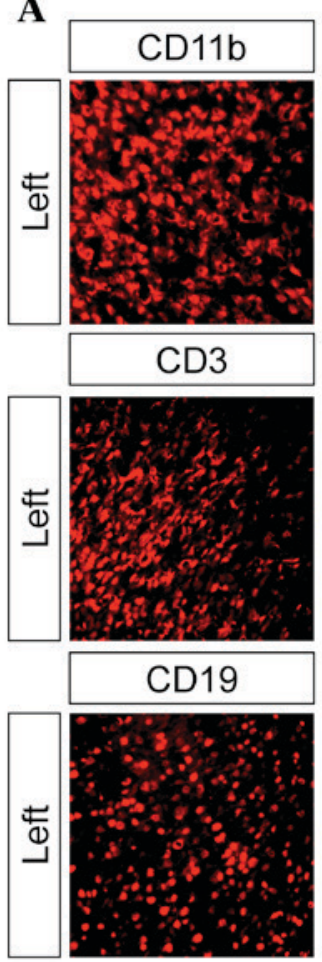

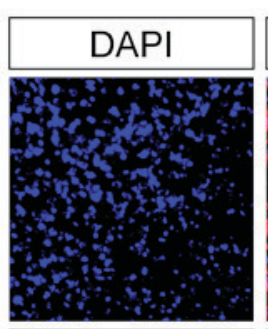

DAPI
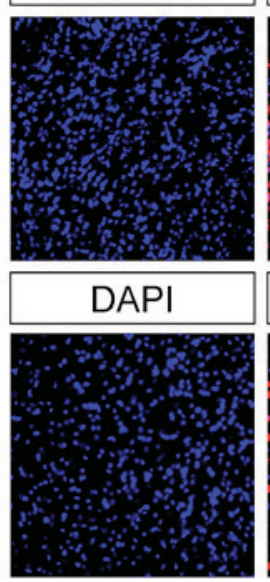

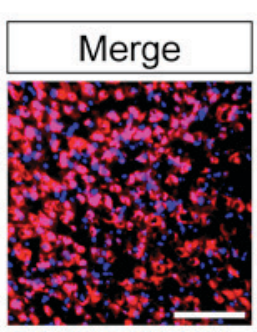

Merge
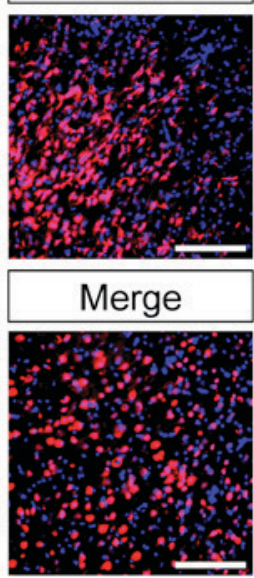

B
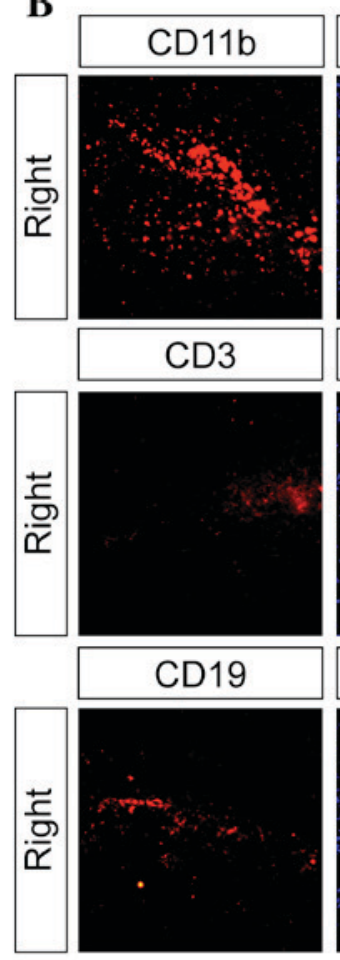
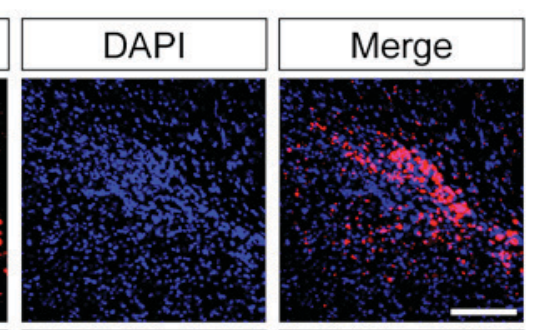

DAPI
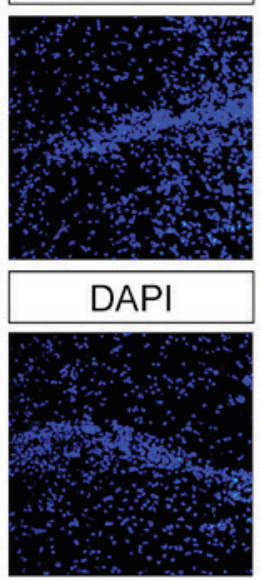

C

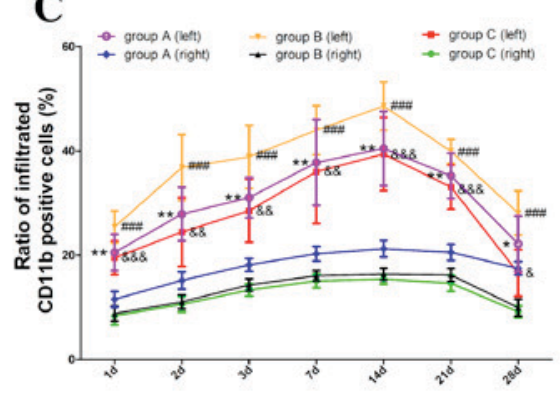

D

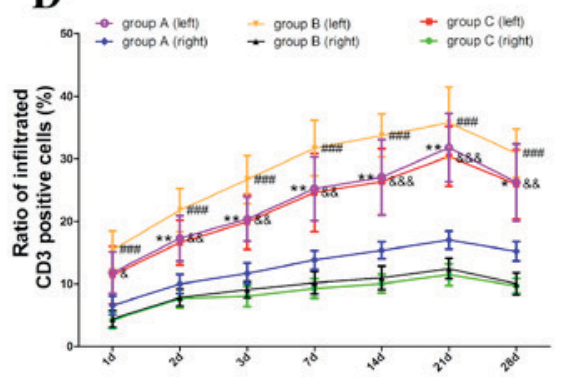

$\mathbf{E}$

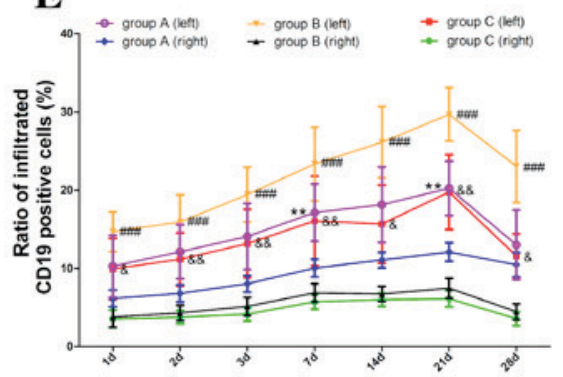

Figure 1. Dynamic changes of infiltrated immune cells. Inflammation secondary to brain injury during skull penetration was determined by immunofluorescence staining. Nuclei were stained with DAPI (blue); microglia/macrophages were identified by anti-CD11b (red); T cells were identified by anti-CD3 (red); and B cells were identified by anti-CD19 (red). In group C, (A) massive infiltrated immune cells, including CD11b-, CD3- and CD19-positive cells, were observed in the left hemisphere; (B) however, only a few immune cells were seen in the right hemisphere at 3 days post-transplantation. (C-E) Statistical analysis of the infiltrated CD11b-, CD3- and CD19-positive cells between the two hemispheres among the three groups are presented, respectively. Data are shown as mean \pm standard deviation $\left(\mathrm{n}=6,{ }^{*} \mathrm{P}<0.05,{ }^{* *} \mathrm{P}<0.01\right.$ vs. group $\mathrm{A}$ (right); ${ }^{\# \#} \mathrm{P}<0.001$ vs. group $\mathrm{B}$ (right); ${ }^{\&} \mathrm{P}<0.05$, \&\& $\mathrm{P}<0.01$, ${ }^{\& \& \&} \mathrm{P}<0.001$ vs. group $\mathrm{C}$ (right), respectively). Scale bar, $100 \mu \mathrm{m}$. DAPI, 4',6-diamidino-2-phenylindole; CD, cluster of differentiation 3; group A, NSCs in the left hemisphere and PBS in the right; group B, NSCs in the right hemisphere and PBS in the left; and group C, equal NSCs in both hemispheres.

Dynamic changes in levels of neurotrophic and immunomodulatory factors. To investigate the effects of transplantation approaches on hosts and grafts, the levels of neurotrophic and immunomodulatory factors in the cerebral cortex were detected (Fig. 2). ELISA results revealed that BDNF, GDNF and NT-3 concentrations in the right hemisphere of group $\mathrm{B}$ and $\mathrm{C}$ increased significantly $(\mathrm{P}<0.05$; Fig. $2 \mathrm{~A}-\mathrm{C}$, respectively), whereas there was no significant difference in BDNF, GDNF and NT-3 concentrations between the two hemispheres in group A. Levels of neurotrophic factors in the NSC transplantation hemispheres were higher than those in the PBS treatment side $(\mathrm{P}<0.05$; Fig. 2A-C).

Furthermore, immunomodulatory factors including proand anti-inflammatory cytokines increased significantly in the left hemisphere in response to the infiltrated immune cells among all the groups $(\mathrm{P}<0.05)$. The levels of pro-inflammatory cytokines (IL-6 and TNF- $\alpha$ ) in the PBS injected hemisphere were significantly higher than those in the NSC transplantation side $(\mathrm{P}<0.05$; Fig. $2 \mathrm{D}$ and $\mathrm{E}$, respectively). However, the levels of anti-inflammatory cytokine IL-10 in the PBS injected hemisphere were significantly lower than that in the NSC transplantation side $(\mathrm{P}<0.05$; Fig. $2 \mathrm{~F})$.

\section{Discussion}

NSC implantation is a promising strategy for neuronal functional recovery in the treatment of CNS diseases $(10,11)$. Although intracerebral transplantation is useful for efficiently delivering NSCs directly, serious limitations to this approach are evident as this form of transplantation may induce brain 
A
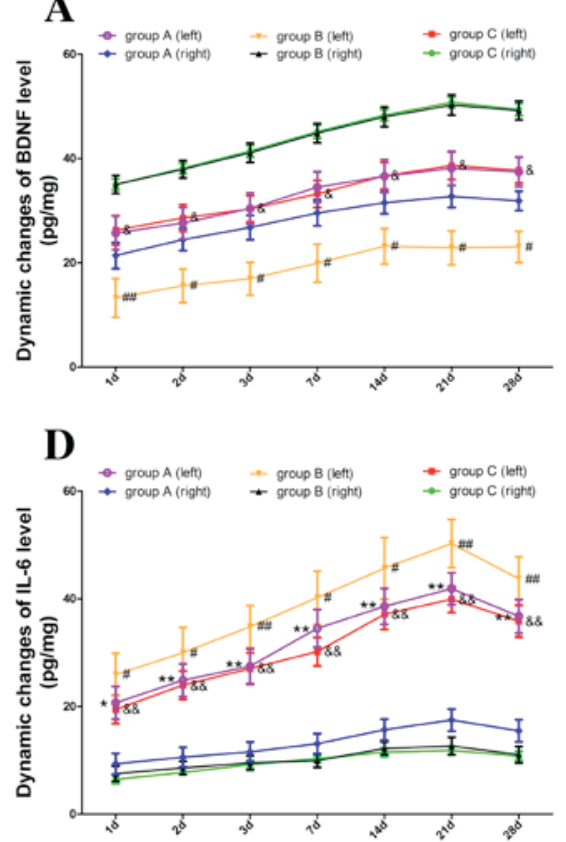

B

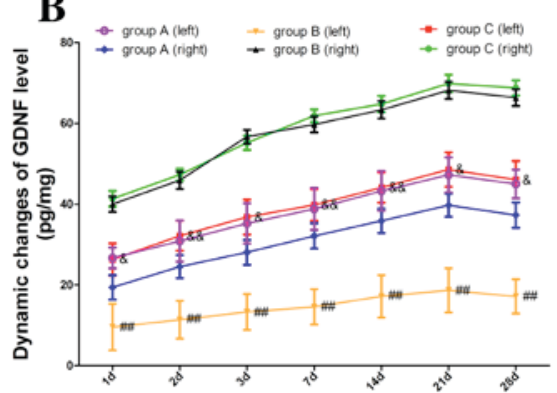

E

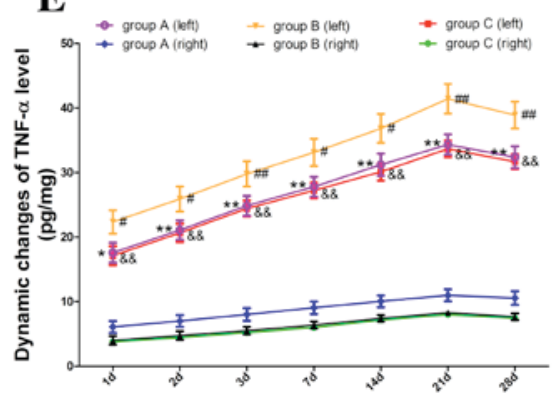

C

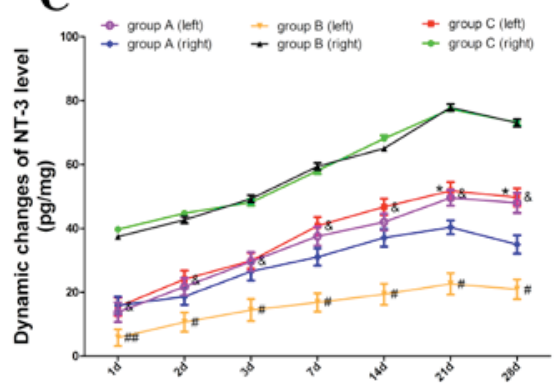

F

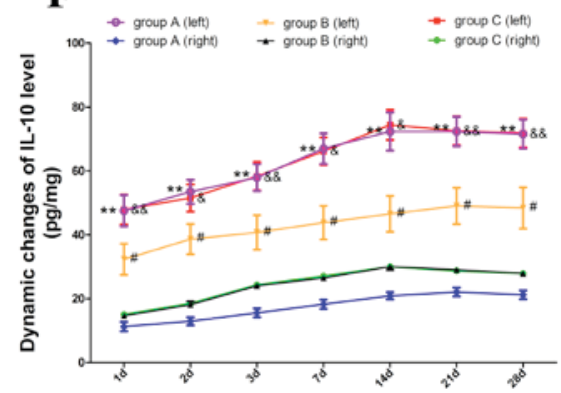

Figure 2. Dynamic changes in levels of neurotrophic and immunomodulatory factors. Levels of neurotrophic and immunomodulatory factors were measured by ELISA assay. (A-C) BDNF, GDNF and NT-3 concentrations in the right hemisphere of group B and C increased significantly, whereas there was no significant difference in BDNF, GDNF and NT-3 concentrations between the two hemispheres in group A. (D and E) Levels of pro-inflammatory cytokines (IL-6 and TNF- $\alpha$ ) in the PBS-injected hemisphere were higher than those in the NSC transplantation side. (F) Level of IL-10 anti-inflammatory cytokine present in the PBS-injected hemisphere was lower than the NSC transplantation hemisphere. Data are shown as mean \pm standard deviation from three independent experiments $\left({ }^{*} \mathrm{P}<0.05,{ }^{* *} \mathrm{P}<0.01\right.$ vs. group A (right); ${ }^{\#} \mathrm{P}<0.05,{ }^{\# \#} \mathrm{P}<0.01$ vs. group B (right); ${ }^{\circledR} \mathrm{P}<0.05$, ${ }^{\&} \mathrm{P}<0.01$ vs. group C (right), respectively). ELISA, enzyme-linked immunosorbent assay; NSC, neural stem cells; BDNF, brain-derived neurotrophic factor; GDNF, glial cell line-derived neurotrophic factor; NT-3, neurotrophin-3; IL-6, interleukin-6; IL-10, interleukin-10; TNF- $\alpha$, tumor necrosis factor alpha; group A, NSCs in the left hemisphere and PBS in the right; group B, NSCs in the right hemisphere and PBS in the left; and group C, equal NSCs in both hemispheres.

injury and secondary inflammation. Previous studies have reported that microtransplantation can improve the function of grafts $(4,5)$; however, the mechanism of how this occurs remains to be elucidated. Few studies have demonstrated that inflammation secondary to brain injuries during skull penetration may also effect the survival and function of NSCs (4-6). In the present study, the effects of two types of intracerebral NSC transplantation approaches on recipients and grafts were compared.

NSCs from B6 embryos that may avoid immune rejection of adult syngeneic mice after transplantation were obtained to ensure the immune cells expressed were predominantly due to secondary inflammation. Microglia and macrophages were the key contributors in the immune response observed, producing cytokines to attract other immune cells and initiating a pro-inflammatory response $(12,13)$. T and $\mathrm{B}$ cell-mediated adaptive immunity also has a complex role in the reconstruction of CNS $(6,14,15)$. The dynamic changes exhibited by immune cells and cytokines revealed that syringe needle skull penetration may reduce inflammation secondary to brain injuries during skull penetration.

Notably, the present study identified that grafted NSCs were able to decrease infiltrated immune cells when compared with PBS-treated groups. The variability in the number of immune cells observed within the group in the left hemisphere indicated that the drill method was less stable. Furthermore, the levels of IL-6, IL-10 and TNF- $\alpha$ in the brains treated with NSCs compared with PBS-treated brains suggested that NSCs may modulate secondary inflammation.
In addition, the elevated levels of BDNF, GDNF and NT-3 in the right hemisphere after NSC transplantation also suggested that syringe needle skull penetration was conducive to the grafts and the recipients when compared with drill penetration.

Although NSC transplantation has been widely used in clinical medicine, this method still exhibits limitations $(3,16)$, including brain injuries and secondary inflammation. Previous studies have shown that the immune system is associated with the effects of NSCs $(6,17)$. Under mild inflammatory conditions, immune cells may promote neurogenesis $(6,18)$. However, under severe inflammatory conditions, immune cells can transform their polarization from anti- to pro-inflammatory, which may result in detrimental effects on neural reconstruction $(19,20)$. Further research should address the association between NSCs and immune cells.

In conclusion, the present study indicated that syringe needle skull penetration is an improved method that results in reduced brain injury and secondary inflammation for intracerebral NSC transplantation, when compared with drill penetration. Furthermore, findings from the present study suggest that NSCs have the potential to modulate inflammation secondary to brain injuries.

\section{Acknowledgements}

The present study was supported by funding from Military Twelfth Five-Year Key Sci-Tech Research Projects (grant no. BWS12J010). 


\section{References}

1. Darsalia V, Allison SJ, Cusulin C, Monni E, Kuzdas D, Kallur T, Lindvall $\mathrm{O}$ and Kokaia Z: Cell number and timing of transplantation determine survival of human neural stem cell grafts in stroke-damaged rat brain. J Cereb Blood Flow Metab 31: 235-242, 2011.

2. English D, Sharma NK, Sharma K and Anand A: Neural stem cells-trends and advances. J Cell Biochem 114: 764-772, 2013.

3. Gage FH and Temple S: Neural stem cells: Generating and regenerating the brain. Neuron 80: 588-601, 2013.

4. Nikkhah G, Cunningham MG, Jödicke A, Knappe U and Björklund A: Improved graft survival and striatal reinnervation by microtransplantation of fetal nigral cell suspensions in the rat Parkinson model. Brain Res 633: 133-143, 1994.

5. Jiang W, Büchele F, Papazoglou A, Döbrössy M and Nikkhah G: Multitract microtransplantation increases the yield of DARPP-32-positive embryonic striatal cells in a rodent model of Huntington's disease. Cell Transplant 20: 1515-1527, 2011.

6. Kokaia Z, Martino G, Schwartz M and Lindvall O: Cross-talk between neural stem cells and immune cells: The key to better brain repair? Nat Neurosci 15: 1078-1087, 2012.

7. Capetian P, Döbrössy M, Winkler C, Prinz M and Nikkhah G: To be or not to be accepted: The role of immunogenicity of neural stem cells following transplantation into the brain in animal and human studies. Semin Immunopathol 33: 619-626, 2011.

8. Schwartz M, Kipnis J, Rivest S and Prat A: How do immune cells support and shape the brain in health, disease, and aging? J Neurosci 33: 17587-17596, 2013.

9. Singec I, Jandial R, Crain A, Nikkhah G and Snyder EY: The leading edge of stem cell therapeutics. Annu Rev Med 58: 313-328, 2007.

10. Lindvall $\mathrm{O}$ and Kokaia Z: Stem cells in human neurodegenerative disorders-time for clinical translation? J Clin Invest 120: 29-40, 2010.
11. Andres RH, Horie N, Slikker W, Keren-Gill H, Zhan K, Sun G, Manley NC, Pereira MP, Sheikh LA, McMillan EL, et al: Human neural stem cells enhance structural plasticity and axonal transport in the ischemic brain. Brain 134: 1777-1789, 2011.

12. Wynn TA, Chawla A and Pollard JW: Macrophage biology in development, homeostasis and disease. Nature 496: 445-455, 2013.

13. Biswas SK and Mantovani A: Macrophage plasticity and interaction with lymphocyte subsets: Cancer as a paradigm. Nat Immunol 11: 889-896, 2010.

14. Ekdahl CT, Claasen JH, Bonde S, Kokaia Z and Lindvall O: Inflammation is detrimental for neurogenesis in adult brain. Proc Nati Acad Sci USA 100: 13632-13637, 2003.

15. Das $S$ and Basu A: Inflammation: A new candidate in modulating adult neurogenesis. J Neurosci Res 86: 1199-1208, 2008.

16. Delcroix GJ, Schiller PC, Benoit JP and Montero-Menei CN: Adult cell therapy for brain neuronal damages and the role of tissue engineering. Biomaterials 31: 2105-2120, 2010.

17. Yirmiya $R$ and Goshen I: Immune modulation of learning, memory, neural plasticity and neurogenesis. Brain Behav Immun 25: 181-213, 2011.

18. Tang Y and Le W: Differential roles of M1 and M2 microglia in neurodegenerative diseases. Mol Neurobiol 53: 1181-1194, 2016.

19. Harry GJ: Microglia during development and aging. Pharmacol Ther 139: 313-326, 2013.

20. Hu X, Leak RK, Shi Y, Suenaga J, Gao Y, Zheng P and Chen J: Microglial and macrophage polarization-new prospects for brain repair. Nat Rev Neurol 11: 56-64, 2015. 